\title{
跨相机交通场景下的车辆空间定位方法
}

\author{
王伟 ${ }^{1,2}$ ，唐心瑶 ${ }^{1}{ }^{*}$, 张朝阳 ${ }^{1)}$, 宋焕生 ${ }^{1)}$, 崔华 1$)$ \\ ${ }^{1)}$ (长安大学信息工程学院 西安 710064) \\ 2) (安徽科力信息产业有限责任公司 合肥 230088) \\ (wangwei_211@chd.edu.cn)
}

\begin{abstract}
摘＼cjkstart要：为解决交通应用中的跨相机全场景车辆空间定位问题，利用单目相机，提出一种全场景透视拼接及车辆 3D 检测相结合的方法. 首先提出一种交通场景下的相机自动标定方法，通过构建相机标定空间模型，自动求取标定参 数并优化; 然后利用公共区域的特征点, 将多相机空间坐标系进行变换统一, 为直观体现全场景物理空间, 将各场 景透视变换至统一的像素-物理坐标系中; 最后, 针对单目视觉下的车辆 $3 \mathrm{D}$ 定位难题, 利用车辆投影的几何约束, 建 立优化模型构建精细化的 3D 包络, 以 3D 包络质心作为车辆空间定位点, 同时可映射至像素-物理坐标系, 体现车辆 的动态信息. 在具有人工特征点的实验环境及无人工特征点的实际应用环境中, 对于跨场景的多类型车辆进行空间 定位实验验证，实验结果表明，该方法可解决交通视频监控中的车辆跨相机大场景下的空间定位难题，在选取的实 验场景中综合定位精度可达到厘米级, 且实时性较好.
\end{abstract}

关键词: 智能交通; 车辆空间定位; 跨相机场景拼接; 自动标定; 3D 包络; 像素-物理坐标系

中图法分类号: TP391.41 DOI: 10.3724/SP.J.1089.2021.18612

\section{Spatial Positioning Method of Vehicle in Cross-Camera Traffic Scene}

\author{
${\text { Wang } \mathrm{Wei}^{1,2)} \text {, Tang Xinyao }}^{1)^{*}}$, Zhang Chaoyang ${ }^{1)}$, Song Huansheng ${ }^{1)}$, and Cui Hua ${ }^{1)}$ \\ 1) (School of Information Engineering, Chang'an University, Xi'an 710064) \\ 2) (Anhui Science and Technology Information Industry Co. Ltd., Hefei 230088)
}

\begin{abstract}
In order to solve the problem of cross-camera and full-scene vehicle spatial positioning in traffic applications, a method combining full-scene perspective stitching and $3 \mathrm{D}$ vehicle detection is proposed. Firstly, a method of automatic camera calibration in traffic scenes is proposed. By building a camera calibration space model, the calibration parameters can be obtained and optimized automatically. Then, feature points in the overlapping area are used to transform and unify the multi-camera spatial coordinate system. In order to intuitively reflect the physical space of the whole scene, the perspective of each scene is transformed into a unified pixel-physical coordinate system. Finally, for the problem of 3D positioning of vehicles under monocular vision, an optimization model is established to construct a refined 3D bounding box by using the geometric constraints of vehicle projection. The $3 \mathrm{D}$ centroid is used as the vehicle spatial positioning point, and the mapping to the pixel-physical coordinate system can reflect the dynamic information of vehicles. In the experimental environment with artificial feature points and the practical application environment without artificial feature points, the spatial positioning experiment is carried out for multi-type ve-
\end{abstract}

收稿日期：2020-08-28; 修回日期：2020-09-29. 基金项目：国家重点研发计划(2019YFB1600500); 陕西省社会发展项目 (2019SF-258); 陕西省科技厅重点研发计划 (2018ZDXM-GY-047); 国家自然科学基金面上项目(6207205); 科技部重大专项 (SQ2019YFB160023). 王伟(1984一), 男, 博士, 讲师, 主要研究方向为计算机视觉、SLAM; 唐心瑶(1996一), 女, 博士研究生, 论文 通讯作者, 主要研究方向为计算机视觉、视频分析; 张朝阳(1984一), 男, 博士, 讲师, 主要研究方向为计算机视觉; 宋焕生(1964一), 男, 博士, 教授, 主要研究方向为计算机视觉; 崔华(1976一), 女, 博士, 教授, 主要研究方向为计算机视觉与人工智能. 
hicles in cross-camera scene. Experimental results show that the algorithm can solve the problem of vehicle positioning in the large cross-camera scene of traffic video surveillance. The comprehensive positioning accuracy in the selected experimental scene can reach the centimeter level with a real-time performance.

Key words: intelligent traffic; vehicle spatial positioning; cross-camera scene splicing; automatic calibration; three-dimensional envelope; pixel-physical coordinate system

近年来，随着智能交通和车路协同技术的发 展，车辆在全场景下准确及时的空间定位具有更 重要的意义 ${ }^{[1]}$. 通过估计周围车辆目标的空间位置, 自动驾驶车辆可以准确地预判和规划自己的行为路 ${ }{ }^{[2]}{ }^{[2]}$; 而在智能交通系统中, 可以进行车辆行为分 析、车流量趋势分析及交通配时控制等进一步的应 用 ${ }^{[3-4]}$. 同时, 在桥梁结构的健康监测中, 车辆的 时空分布是桥梁荷载空间模型建立的基础, 而车 辆在桥梁全场景空间的定位是其关键技术之一. 当前对于车辆在场景中的物理定位大都采用激光 雷达或全球定位系统(global positioning system, GPS)等方式 ${ }^{[5-7]}$, 然而激光雷达等方式的定位范围 较小, 很难做到全场景的定位, 且成本较高, 并未 在道路交通中大规模普及; 使用 GPS 等方式也具 有一定的限制. 首先, GPS 并未在大部分车辆中普 及, 且因隐私等问题, 数据较难获取; 其次, 在隧 道等非露天环境下, GPS 信号会变弱. 随着视频监 控设备在道路场景中的普及, 基于监控相机的车 辆全场景空间定位成为了可靠的选择之一. 然而 当前主流研究对于车辆的跟踪定位大都基于单场 景, 对于大范围跨相机场景的车辆定位研究较少; 同时, 如何从单目视觉中精确求取车辆的物理定 位也是研究难点之一. 因此, 本文涉及的技术难点 分为 2 个方面: 跨相机交通场景像素-物理拼接以 及单目视觉下的车辆 $3 \mathrm{D}$ 包络构建. 下面对相关研 究进行简要综述.

所谓全场景的像素-物理拼接，是指所拼接的 场景不仅包含原场景的像素信息，同时也包含物 理尺度信息. 当前对于多场景的拼接方法主要分 为 2 类, 一类是基于图像配准的图像拼接 ${ }^{[8]}$, 思路 为将具有重叠区域的小视角、低分辨率的多幅图 像, 检测出多个相对应的局部不变描述子(如 SIFT, SURF 特征算子), 并将这些局部不变描述子进行 特征匹配构建透视单应矩阵(homography matrix), 最后基于匹配点进行透视变换, 将多幅图像进行 拼接, 形成一幅具有高分辨率、宽视角的全景图像. 该类方法研究成熟, 应用也较广泛, 尤其在手机摄
像全景拼接应用效果良好 ${ }^{[9]}$. 但该类方法并没有用 到相机的标定, 无法在拼接后的图像中体现物理信 息. 另一类方法是基于相机标定的图像拼接 ${ }^{[10-11]}$, 对于有些大幅面物体, 可以拍摄多幅图像, 每幅图 像覆盖物体的不同部分, 同时保证相机经过标定 并且与确定的世界坐标系之间的相对关系已知， 就可以将多幅图像通过统一透视变换拼接为覆盖 整个物体的大图. 此时获得的图像为标准的像素物理拼接图像, 可在其中进行世界坐标的测量与 定位. 该类方法需要手动标定各个相机, 操作较复 杂, 大多应用于零部件的小视角物体的测量, 鲜有 针对道路等大视角场景的测量. 在本文团队前期 的工作中, 关于道路场景的拼接进行了一定的研 究 ${ }^{[12]}$, 但早期研究并没有对跨相机大场景的累积误 差做矫正，同时也没有实现车辆目标的空间定位.

车辆的空间定位可看做车辆空间质心求取, 即车辆 $3 \mathrm{D}$ 包络构建的问题. 由于射影几何学的限 制, 单视图无法直接获取每个点的深度信息, 只能 根据投影信息进行估计, 当前的单目视觉下的车 辆 $3 \mathrm{D}$ 估计主要分为 2 类. 第 1 类是基于几何约束 的目标 3D 估计 ${ }^{[13-16]}$, 其主要基于灭点约束进行 3D 包络构建. 例如, Sochor 等 ${ }^{[17]}$ 和 Yang 等 ${ }^{[18]}$ 均采用 3 个垂直方向灭点约束获取目标 $3 \mathrm{D}$ 包络, 然后基于 先验知识获取尺度信息, 继而得到目标的空间形 态. 这类方法只利用了几何约束, 因此处理速度 快, 但多个灭点的估计在一定视角下并不稳定, 因 此具有一定的局限性. 第 2 类方法是基于深度学习 的目标 $3 \mathrm{D}$ 估计. 近年来, 利用深度学习估计车辆 $3 \mathrm{D}$ 信息的方法得到快速发展 ${ }^{[19-20]}$. 例如, $\mathrm{Xu}$ 等 ${ }^{[21]}$ 提出一种估计点云深度图的模型, 但其计算过于 奢侈. 为了兼顾效率，一些学者采用了深度学习与 几何约束相结合的方式, 如百度 Apollo 引用的方

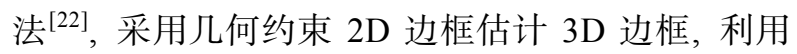
卷积神经网络(convolutional neural networks, CNN) 模型首先估计物体 $3 \mathrm{D}$ 朝向, 然后回归物体尺寸和 3D 中心, 最后得到物体的整个姿态和位置. 然而, 此类方法依赖于大量 3D 标注的精度, 此外, 深度 
网络耗时较大, 较难满足实时应用.

综上所述，本文在分析了国内外相关研究优 缺点的基础上，提出了基于像素-物理拼接的跨场 景下车辆空间定位方法. 首先, 针对单交通场景构 建自标定模型，对各场景空间坐标系进行旋转平 移变换，统一全场景的空间坐标系，并利用虚线标 识进行累积误差校正; 然后, 对统一空间坐标系的 全场景构建带尺度因子的俯视图, 并用原场景像 素进行填充; 最后, 将参数空间内的车辆 $3 \mathrm{D}$ 包络 在图像上进行投影，构建投影与车辆轮廓的几何 约束, 进行优化确定精确的质心, 并将质心变换至 统一空间坐标系得到其空间定位. 在搭建的实验 和实际场景中进行实验及结果分析, 从而验证本 文方法的有效性.

\section{1 跨相机道路区间像素-物理全景拼接}

\section{1 道路场景下相机标定空间模型}

当前道路场景的标定大都基于手工标定的方 式, 而目前流行的监控相机大都是可变焦和旋转 的云台相机，传统手工标定方式并不适合多变的 道路场景. 因此，在本文前期的工作中，对道路场 景中的相机自动标定模型进行了研究 ${ }^{[23]}$. 图 1 所 示为道路单场景中的相机空间模型示意图.

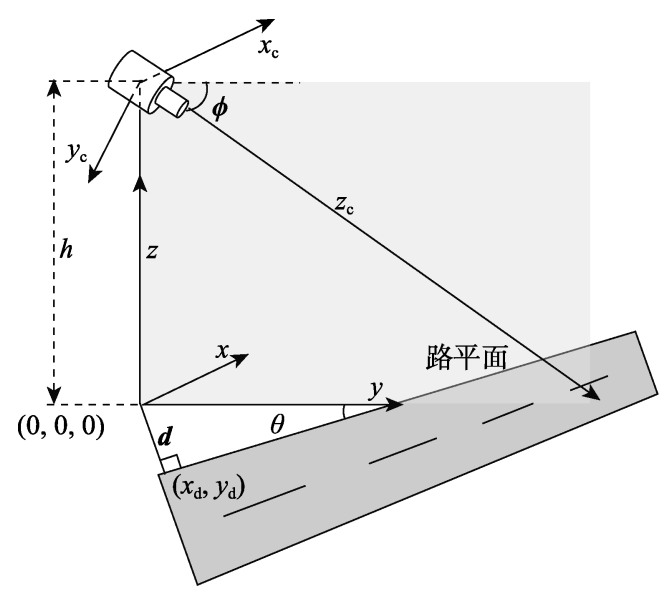

图 1 道路单场景中相机空间模型示意图

此模型中, 设相机焦距为 $f$, 原点距离地面高 度为 $h$, 相对于路面相机俯仰角为 $\phi$, 偏转角为 $\theta$, 其中世界坐标系的 $x y$ 平面与路面重合，世界坐标 原点为相机原点在路面的投影点， $z$ 轴与地面垂直. 考虑空间坐标点的齐次表示 $\boldsymbol{p}_{\text {world }}=[x, y, z, 1]^{\mathrm{T}}$, 在 图像坐标系齐次表示为 $\boldsymbol{p}_{\text {image }}=[\alpha u, \alpha v, \alpha]^{\mathrm{T}}, \alpha \neq 0$ 为尺度因子，可得投影方程为

$$
\left[\begin{array}{r}
\alpha u \\
\alpha v \\
\alpha
\end{array}\right]=\left[\begin{array}{rrrr}
f & 0 & 0 & 0 \\
0 & -f \sin \phi & -f \cos \phi & f h \cos \phi \\
0 & \cos \phi & -\sin \phi & h \sin \phi
\end{array}\right]\left[\begin{array}{l}
x \\
y \\
z \\
1
\end{array}\right]=\boldsymbol{P}\left[\begin{array}{l}
x \\
y \\
z \\
1
\end{array}\right]
$$

其中, 标定参数 $f, \phi, h$ 和 $\theta$ 可由道路标识线、道路 宽度和灭点等几何信息等价表示, 并在道路中存 在多条几何标识约束的条件下可对标定参数进行 优化, 推导过程详见文献 [23]. 同时, 参考文献 [24]的推导, 易得原点与道路边界的垂点, 设为向 量 $\boldsymbol{d}=\left(x_{\mathrm{d}}, y_{\mathrm{d}}\right)$. 简化式(1)可得

$$
\left\{\begin{array}{l}
u=\frac{\alpha u}{\alpha}=\frac{f x}{y \cos \phi-z \sin \phi+h \sin \phi} \\
v=\frac{\alpha v}{\alpha}=\frac{f h \cos \phi-f y \sin \phi-f z \cos \phi}{y \cos \phi-z \sin \phi+h \sin \phi}
\end{array}\right.
$$

由式(2)可知, 当给定目标高度 $z$ 已知时, 也 可获得图像点坐标至世界坐标的逆投影点.

\section{2 统一坐标系及全景拼接}

在第 1.1 节中的各场景相机标定完成后，事实 上已建立起各场景的图像与道路平面 $(z=0)$ 的像 素-物理映射关系，而由于各场景世界坐标系相异， 需要将坐标系进行统一才能做有效测量, 本文将 首先统一至第 1 个场景世界坐标系. 为了获得相邻 坐标系的空间变换关系, 设计了公共视野中路面 特征点组, 如图 2 所示.

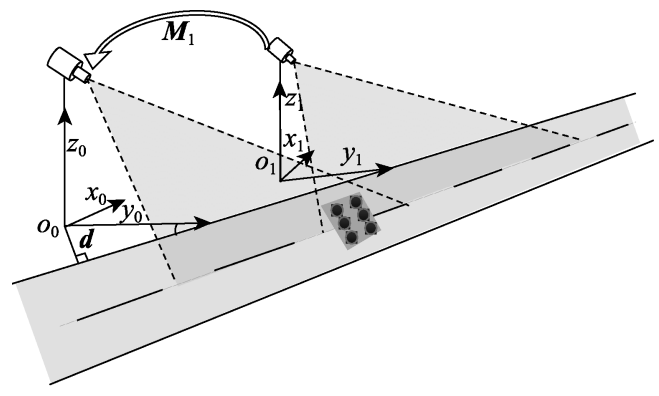

图 2 跨相机道路区间示意图

以坐标系 $o_{0}-x_{0} y_{0} z_{0}$ 和 $o_{1}-x_{1} y_{1} z_{1}$ 为例, 假设有 $n$ 个特征点, 且在 2 个场景图像中的像素坐标已 知, 通过代人式(2)可求得其物理坐标, 结果为

$$
\left\{\begin{array}{l}
\left\{P_{0 i}=\left(x_{0 i}, y_{0 i}, 0\right) \mid 1 \leqslant i \leqslant n\right\} \\
\left\{P_{1 i}=\left(x_{1 i}, y_{1 i}, 0\right) \mid 1 \leqslant i \leqslant n\right\}
\end{array}\right.
$$

其中, $P_{0 i}$ 表示第 $i$ 个特征点在 $o_{0}-x_{0} y_{0} z_{0}$ 的物理坐 标; $P_{1 i}$ 表示第 $i$ 个特征点在 $o_{1}-x_{1} y_{1} z_{1}$ 的物理坐标. 由坐标系空间坐标变换原理可知, 特征点在 2 个坐 标系坐标的变换关系为 


$$
\left[\begin{array}{rrr}
x_{01} & \cdots & x_{0 n} \\
y_{01} & \cdots & y_{0 n} \\
0 & \cdots & 0 \\
1 & \cdots & 1
\end{array}\right]=\left[\begin{array}{rr}
\boldsymbol{R}_{1} & \boldsymbol{T}_{1} \\
0 & 1
\end{array}\right]\left[\begin{array}{rrr}
x_{11} & \cdots & x_{1 n} \\
y_{11} & \cdots & y_{1 n} \\
0 & \cdots & 0 \\
1 & \cdots & 1
\end{array}\right]
$$

其中, $\boldsymbol{R}_{1}$ 为 2 个坐标系的旋转矩阵; $\boldsymbol{T}_{1}$ 为平移向 量. 为了表示方便, 引人空间坐标变换矩阵

$$
\boldsymbol{M}_{1}=\left[\begin{array}{rr}
\boldsymbol{R}_{1} & \boldsymbol{T}_{1} \\
0 & 1
\end{array}\right]
$$

由于本文构建的标定模型使各场景的 $x y$ 坐标 平面均与道路平面重合, 空间坐标系的旋转变换 只需一个绕 $z$ 轴角度即可; 同时, 平移向量也只需 2 个参数 $(z=0)$. 因此, 理论上只需 2 个特征点即 可解得空间变换矩阵，多余的特征点可用于最小 二乘法参数优化.

由标定、镜头畸变和空间坐标变换等产生的误 差，使拼接后跨场景之间存在一定的累计误差. 若 所跨的场景较多，这种累计误差将会使定位精度 变差, 因此, 本文将利用相邻场景的几何标识进行 校正. 如图 3 所示, 在相邻 2 个场景视野中, 假设 初始车道线起始点像素坐标已知, 可联合式(2)(4) 求得其在统一坐标系下的坐标, 设为 $\left(x_{01}, y_{01}, 0\right)$ 和 $\left(x_{11}, y_{11}, 0\right)$, 而道路中虚线尺寸满足一定的国家标 准，为已知值，因此根据 2 个起始点间隔多少条车 道线可以计算出实际的距离. 设场景 2 中真实距离 的初始车道线坐标为 $\left(x_{11}^{\prime}, y_{11}^{\prime}, 0\right)$, 则可得修正的平 移向量为 $\boldsymbol{T}_{1}^{\prime}=\left(x_{11}^{\prime}-x_{11}, y_{11}^{\prime}-y_{11}, 0\right)^{\mathrm{T}}$. 因此修正之 后空间坐标变换矩阵为

$$
\boldsymbol{M}_{1}^{\prime}=\left[\begin{array}{rr}
\boldsymbol{R}_{1} & \boldsymbol{T}_{1}-\boldsymbol{T}_{1}^{\prime} \\
0 & 1
\end{array}\right]
$$

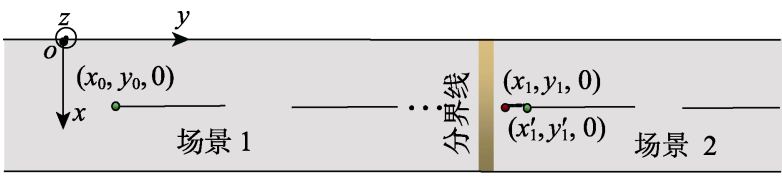

图 3 统一世界坐标空间的拼接结果示意图

为了更直观、方便地表示道路物理区间，本文 再将统一世界坐标系进行空间变换，如图 3 所示， 将图 2 中的原点平移至 $o_{0}$ 在道路实线的垂点 $O$, $y_{0}$ 轴旋转至与道路平行方向 $y$ 轴, 并将 $x$ 轴正负 方向翻转, 此时, 道路方向物理坐标沿 $y$ 轴递增, 且道路宽度物理坐标沿 $x$ 轴递增, 将此坐标系设 为最终的统一世界坐标系, 设为 $o-x y z$. 由图 1 可 知, 通过建立的空间标定几何模型, 可得到绕 $z$ 轴
旋转参数 $\theta$ 和原点平移向量 $\boldsymbol{d}$. 由空间坐标系变 换原理可知, $o_{0}-x_{0} y_{0} z_{0}$ 至 $o-x y z$ 的空间坐标变换 矩阵为

$$
\boldsymbol{M}_{0}=\left[\begin{array}{rrrr}
\cos \theta & -\sin \theta & 0 & -x_{\mathrm{d}} \\
\sin \theta & \cos \theta & 0 & -y_{\mathrm{d}} \\
0 & 0 & 1 & 0 \\
0 & 0 & 0 & 1
\end{array}\right]\left[\begin{array}{cccr}
-1 & 0 & 0 & 0 \\
0 & 1 & 0 & 0 \\
0 & 0 & 1 & 0 \\
0 & 0 & 0 & 1
\end{array}\right]
$$

下面将构建任意单场景的空间坐标系至统一 坐标系的变换关系, 设第 $n$ 个道路场景中的任意 空间点 $\left(x_{n}, y_{n}, z_{n}\right)$ 在统一世界坐标 $o-x y z$ 中表示为 $\left(x_{n}^{o}, y_{n}^{o}, z_{n}^{o}\right)$, 根据式(6)(7), 级联相乘相邻空间坐标 系变换, 并最后乘以 $\boldsymbol{M}_{0}$, 可得

$$
\left[\begin{array}{r}
x_{n}^{o} \\
y_{n}^{o} \\
z_{n}^{o} \\
1
\end{array}\right]=\boldsymbol{M}_{n}^{\prime} \boldsymbol{M}_{n-1}^{\prime} \cdots \boldsymbol{M}_{1}^{\prime} \boldsymbol{M}_{0}\left[\begin{array}{c}
x_{n} \\
y_{n} \\
z_{n} \\
1
\end{array}\right]
$$

为了更直观地体现拼接的结果, 以及方便在 全景图中显示定位位置, 本文建立了像素-物理全 景拼接图，具体过程如下:

Step1. 基于尺度因子 $\lambda$ (单位为 $\mathrm{cm} / \mathrm{pixel}$, 为超参 数可调节)建立一幅空白图像 $I$, 图像分辨率可由道路 全景长和宽物理距离决定.

Step2. 遍历 $I$, 对于像素点 $\left(u_{\mathrm{p}}, v_{\mathrm{p}}\right)$, 其对应的道路 映射物理点为 $\left(\lambda u_{\mathrm{p}}, \lambda v_{\mathrm{p}}, 0\right)$, 判断其所在的分场景, 并代 人式(8)求逆, 获取其单场景坐标系下的物理坐标; 并根 据该场景的标定信息, 将该物理坐标代人式(2), 获取对 应的像素点 $\left(u_{\mathrm{p}}^{\prime}, v_{\mathrm{p}}^{\prime}\right)$.

Step3. 将 $\left(u_{\mathrm{p}}^{\prime}, v_{\mathrm{p}}^{\prime}\right)$ 赋值给 $\left(u_{\mathrm{p}}, v_{\mathrm{p}}\right)$, 转 Step2, 直至该 像素-物理全景拼接图构建完毕.

图 4 所示为像素-物理全景拼接图的部分展示.

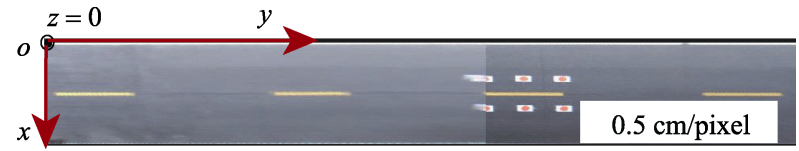

图 4 像素-物理全景拼接的部分展示

\section{2 基于车辆 3D 包络质心的空间定位}

\section{1 基于灭点约束的车辆 3D 包络模型构建}

为了求取车辆的质心物理坐标, 需要先对图像 中的车辆目标进行 $3 \mathrm{D}$ 包络(长方体盒), 而由于单目 视觉本身的限制, 无法直接在图像中获取深度信息, 因此本文将通过几何约束的方式进行估计. 
在透视几何学中, 灭点是重要的几何参数, 在 道路场景中, 道路方向灭点较容易获得, 如道路边 缘、车道线和车辆轨迹直线等都平行于道路方向, 其在图像中的投影交点即为灭点 ${ }^{[25]}$. 当车辆沿道 路方向行驶时，根据透视投影原理，理论上车辆刚 体的 3D 包络约束于灭点形成的四棱雉上, 设 $3 \mathrm{D}$ 包络的 8 个顶点物理坐标为 $P_{1}, P_{2}, \cdots, P_{8}$, 对应图像 坐标为 $p_{1}, p_{2}, \cdots, p_{8}$, 如图 5 所示. 基于图 1 建立的 相机空间标定模型，由文献 [24]推出齐次坐标 $P_{0}=(-\tan \theta, 1,0,0)$ 可表示灭点的物理点. 由灭点的 约束，易知 $P_{1} P_{0}$ 的方向向量为 $\boldsymbol{d}_{1}=(-\sin \theta, \cos \theta, 0)$, 而 $P_{1} P_{0}$ 的方向与 $P_{1} P_{2}$ 垂直, 因此其方向向量为 $\boldsymbol{d}_{\mathrm{w}}=(\cos \theta, \sin \theta, 0)$, 同时，垂直方向的方向向量可 表示为 $\boldsymbol{d}_{\mathrm{h}}=(0,0,1)$. 假设车辆长宽高 $\left(l_{\mathrm{v}}, w_{\mathrm{v}}, h_{\mathrm{v}}\right)$ 已 知, 根据 $3 \mathrm{D}$ 包络框顶点相对关系, 基于 $P_{1}$ 点即可 推导出 $3 \mathrm{D}$ 包络的 8 个顶点物理坐标为

$$
\left[\begin{array}{ll}
P_{1} & P_{2} \\
P_{3} & P_{4} \\
P_{5} & P_{6} \\
P_{7} & P_{8}
\end{array}\right]=\left[\begin{array}{rr}
\left(x_{1}, y_{1}, 0\right) & P_{1}+w_{\mathrm{v}} \boldsymbol{d}_{\mathrm{w}} \\
P_{2}+l_{\mathrm{v}} \boldsymbol{d}_{1} & P_{1}+l_{\mathrm{v}} \boldsymbol{d}_{1} \\
P_{1}+h_{\mathrm{v}} \boldsymbol{d}_{\mathrm{h}} & P_{2}+h_{\mathrm{v}} \boldsymbol{d}_{\mathrm{h}} \\
P_{3}+h_{\mathrm{v}} \boldsymbol{d}_{\mathrm{h}} & P_{4}+h_{\mathrm{v}} \boldsymbol{d}_{\mathrm{h}}
\end{array}\right]
$$

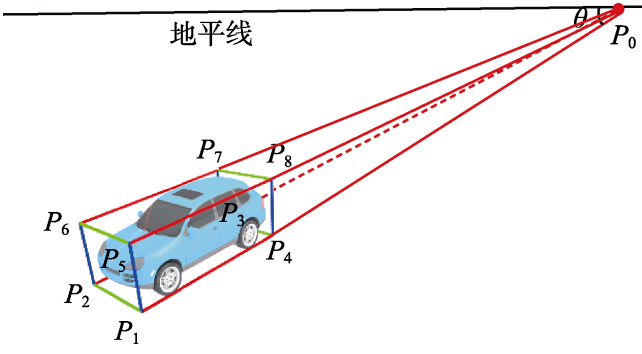

图 5 车辆 $3 \mathrm{D}$ 包络的灭点几何约束示意图

\section{2 基于轮廓约束的车辆 3D 包络优化求解}

由第 2.1 节可知，在车辆尺寸已知的情况下， 结合标定信息, 只需一个起始点即可构建出车辆 的 3D 包络. 而实际应用中, 车辆的尺寸信息并不 易获得，因此本节将利用投影轮廓约束对车辆尺寸 进行估算. 基于第 2.1 节的分析, 3D 包络的构建可 转化为求解车辆长宽高 $\left(l_{\mathrm{v}}, w_{\mathrm{v}}, h_{\mathrm{v}}\right)$ 及顶点 $P_{1}$ 的坐标, 由于在已知标定的条件下, $P_{1}$ 点物理坐标和像素坐 标可以相互转换，设 $P_{1}$ 投影像素坐标为 $(u, v)$, 因 此, 问题转化为参数向量 $\boldsymbol{V}=[w, l, h, u, v]^{\mathrm{T}}$ 的估计.

由于车辆是类长方体的刚体, $3 \mathrm{D}$ 包络构建越 准确, 其投影与图像中车辆轮廓越重合, 因此, 本 文利用这种轮廓的几何约束估计参数向量 $\boldsymbol{V}$. 关 于车辆轮廓的提取, 既可采用传统的混合高斯模
型(Gaussian mixture model, GMM)对运动目标进行 前景分割，也可利用近年来流行的 Mask R-CNN ${ }^{[26]}$ 等深度网络提取, 本文采用第 2 种方法提高检测精 度. 如图 6a 所示, 设提取出的图像中车辆轮廓为 $C$, 参数向量 $\boldsymbol{V}$ 对应的 $3 \mathrm{D}$ 包络在图像中的投影凸 包为 $C_{\mathrm{v}}$, 优化的目的是使投影 $C_{\mathrm{v}}$ 与车辆轮廓 $C$ 差 值最小. 设 $\boldsymbol{V}_{1}=\left[w_{1}, l_{1}, h_{1}, u_{1}, v_{1}\right]^{\mathrm{T}}$ 为一组给定的初 始参数向量, 构造对应的投影凸包与车辆外轮廓 的差值约束函数, 具体步骤如下.

Step1. 将 $p_{1}=\left(u_{1}, v_{1}\right)$ 代人式(2)即可得到对应的物 理点 $P_{1}=\left(x_{1}, y_{1}, 0\right)$, 并将 $\left(l_{1}, w_{1}, h_{1}\right)$ 联合 $P_{1}$ 代人式 $(9)$, 可 得到 $3 \mathrm{D}$ 包络的 8 点物理坐标.

Step2. 将 3D 包络的 8 点物理坐标代人式(2), 可反 求出 8 个投影点并求凸包, 获得某组已知 $\boldsymbol{V}_{1}$ 对应的 $3 \mathrm{D}$ 包 络投影凸包，设为 $\left\{p_{i} \mid 1 \leqslant i \leqslant m\right\} ， m$ 为凸包的顶点数量.

Step3. 为了更细化地描述差值约束, 在相邻的投 影凸包顶点进行等间隔插值插人 $v$ 个新顶点, 因此投影 凸包的稠密顶点表示为 $\left\{p_{i} \mid 1 \leqslant i \leqslant m(v+1)\right\}$.

Step4. 求轮廓 $C$ 的重心 $O$, 连接 $O p_{i}$ 获得与 $C$ 的 交点 $q_{i}$, 则约束误差为 $\sum_{j=1}^{m(v+1)} p_{i} q_{i}$.

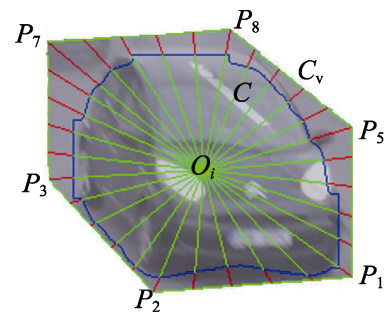

a. 3D包络示例

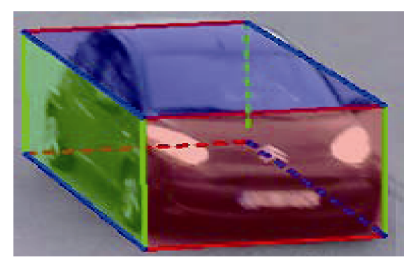

b. 投影轮廓约束
图 6 基于轮廓约束的车辆 3D 包络估计图

图 6a 所示为 $v=4$ 时对应的凸包与车辆轮廓的 差值, 用红色线段直观表示, 另外 $p_{1}, p_{2}, p_{3}$, $p_{7}, p_{8}, p_{5}$ 为投影凸包顶点. 构建约束函数为

$$
\left\{\begin{aligned}
\min _{V} & \sum_{j=1}^{m(v+1)} \frac{p_{i} q_{i}}{m(v+1)} \\
\text { s.t. } & w_{0} \leqslant w_{\mathrm{v}} \leqslant w_{1}, l_{0} \leqslant l_{\mathrm{v}} \leqslant l_{1}, \\
& h_{0} \leqslant h_{\mathrm{v}} \leqslant h_{1},\left(u_{1}, v_{1}\right) \in R
\end{aligned}\right.
$$

其中, $\left(w_{0}, w_{1}\right),\left(l_{0}, l_{1}\right)$ 和 $\left(h_{0}, h_{1}\right)$ 为车辆长宽高的 约束范围, Mask-RCNN 可识别出粗略的车(如轿 车、卡车和公交车等), 不同的车型有不同的取值 范围; $R$ 为针点 $\left(u_{1}, v_{1}\right)$ 的取值范围, 可设在轮廓周 边. 设定完取值范围之后, 将缩小参数空间, 减少 最后参数的搜索时间, 本文采用最小二乘优化方 法迭代求取最优的参数向量. 图 $6 \mathrm{~b}$ 所示为最优参 
数向量 $\boldsymbol{V}$ 对应的 $3 \mathrm{D}$ 包络.

最后求取该 $3 \mathrm{D}$ 包络的质心物理坐标 $P_{\text {centroid }}=\frac{1}{2}\left(P_{1}+P_{7}\right)$, 并代人式(8) 获得其在统一坐 标系中的坐标，同时在乘以尺度因子 $\lambda$ 之后，可在 像素-物理坐标系中进行直观显示.

\section{3 实验结果及分析}

公开的数据集中直接针对跨相机场景进行车 辆空间定位的数据比较少, 因此本文设计 2 类场景 进行实验和实际验证. 实验场景验证方案 1 具有人 工设计特征点, 场景环境较为简单, 且实验车辆长 宽高物理尺寸已知; 实际场景验证方案 2 为实际道 路场景采集的无人工特征点的验证方案，同时场 景环境较为复杂, 定位的车辆长宽高未知. 本文的 实验环境计算机配置如下: Windows 10 系统, $8 \mathrm{~GB}$ 内存, Intel $2.50 \mathrm{GHz} \mathrm{CPU}$, 英伟达 GTX1060Ti 显 卡, 单场景采用 SONY HDR-CX405 型号相机进行 拍摄, 有效像素为 229 万, 物理焦距范围为 1.9 57.0 mm. 下面将对这 2 类场景验证结果进行 分析.

\section{1 实验场景验证}

图 7a 和图 7b 所示为在实验道路现场布设的跨

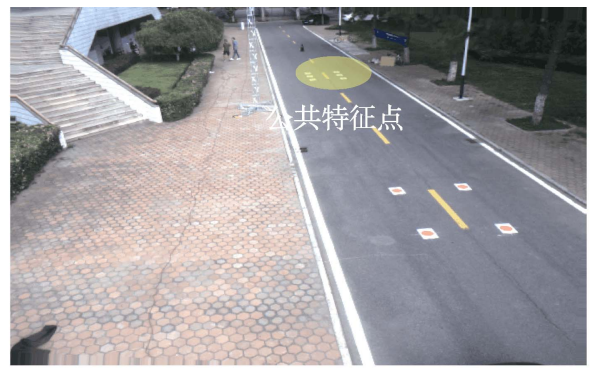

a. 场景1

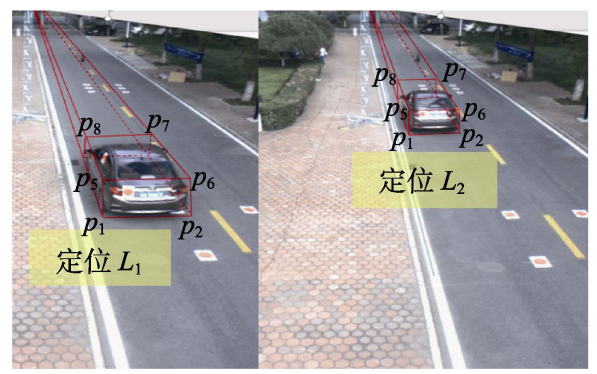

c. 场景1车辆定位

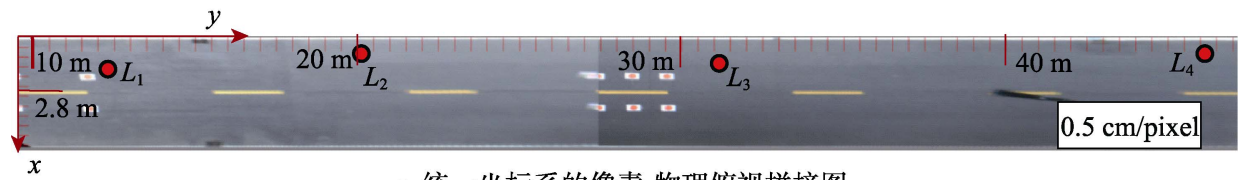

e. 统一坐标系的像素-物理俯视拼接图

图 7 实验场景下验证结果
相机场景, 其中黄色阴影部分为手工设置的公共 区域特征点, 为了优化求取空间变换矩阵, 特设置 了 6 个圆形标识. 在该实际道路场景中, 道路虚线 长度为 $2.0 \mathrm{~m}$, 道路宽度为 $5.6 \mathrm{~m}$, 基于道路相机标 定空间模型, 可解得标定参数及投影矩阵, 如表 1 前 5 列所示. 然后利用公共区域 6 个特征点，根据 第 1.2 节的方法求取并校正 2 个场景空间坐标变换 矩阵得到 $\boldsymbol{M}_{1}^{\prime}$, 同时求取出场景 1 至统一世界坐标 $o-x y z$ 的变换矩阵 $\boldsymbol{M}_{0}$, 场景 2 至 $O-x y z$ 的变换矩 阵则为 $\boldsymbol{M}_{1}^{\prime} \boldsymbol{M}_{0}$, 如表 1 最后 1 列所示. 最后基于本 文的像素-物理全景拼接图构建方法, 根据各场景 建立的统一空间坐标变换矩阵、投影矩阵和投影像 素, 构建跨相机场景像素-物理全景拼接图, 如图 $7 \mathrm{e}$ 所示.

实验过程中，在跨相机场景中驾驶一辆已知 尺寸(长/宽/高: $4.2 \mathrm{~m} / 1.6 \mathrm{~m} / 1.4 \mathrm{~m}$ )的轿车驶过, 如 图 7c 和图 7d 所示, 随机在 2 个场景中各选取 2 个 车辆定位位置进行验证(随机选取的位置在实验场 景中可人工标出其实际位置和坐标), 并将 4 个空 间定位记为 $\left\{L_{i} \mid 1 \leqslant i \leqslant 4\right\}$, 由于车辆尺寸已知, 因 此可只利用第 2.1 节中的灭点约束方法构建其 3D 包络, 并求取质心. 最后通过统一空间坐标变换矩 阵求取其在统一空间坐标系下的物理坐标, 并在

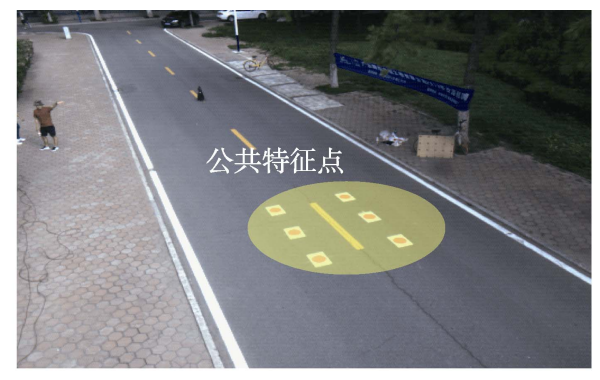

b. 场景 2

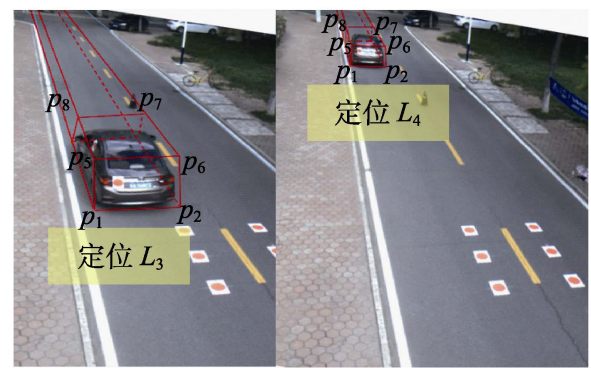

d. 场景2车辆定位 
表 1 实验场景下的标定结果及统一空间坐标变换矩阵

\begin{tabular}{|c|c|c|c|c|c|c|c|c|c|c|c|c|c|c|}
\hline 场景 & $f$ & $\phi / \mathrm{rad}$ & $\theta / \mathrm{rad}$ & $h / \mathrm{m}$ & \multicolumn{4}{|c|}{ 投影矩阵 $\boldsymbol{P}$} & \multicolumn{6}{|c|}{ 统一空间坐标变换矩阵 } \\
\hline 1 & 1835.66 & 0.44 & 0.11 & 5.26 & $\left(\begin{array}{c}1835.66 \\
0 \\
0\end{array}\right.$ & $\begin{array}{l}0 \\
-7.84 \times 10^{2} \\
0.904\end{array}$ & $\begin{array}{l}0 \\
-1.66 \times 10^{3} \\
-0.427\end{array}$ & $\left.\begin{array}{l}0 \\
8.73 \times 10^{6} \\
2.25 \times 10^{3}\end{array}\right)$ & $\left(\begin{array}{l}-0.994 \\
0.110 \\
0 \\
0\end{array}\right.$ & $\begin{array}{c}-0.11 \\
0.99 \\
0 \\
0\end{array}$ & $\begin{array}{lll}10 & 0 & \\
94 & 0 & - \\
& 1 \\
& 0\end{array}$ & $\begin{array}{c}257.3 \\
-13.2 \\
0 \\
1\end{array}$ & $\begin{array}{l}398 \\
233 \\
\end{array}$ & \\
\hline 2 & 1812.01 & 0.48 & 0.41 & 5.23 & $\left(\begin{array}{c}1812.01 \\
0 \\
0\end{array}\right.$ & $\begin{array}{l}0 \\
-2.67 \times 10^{2} \\
0.887\end{array}$ & $\begin{array}{l}0 \\
-1.61 \times 10^{3} \\
-0.462\end{array}$ & $\left.\begin{array}{l}0 \\
8.41 \times 10^{6} \\
2.42 \times 10^{3}\end{array}\right)$ & $\left(\begin{array}{l}2.02 \times 1 \\
-6.151 \\
0.349 \\
0\end{array}\right.$ & ${ }^{10^{3}}$ & $\begin{array}{r}1.21 \times \\
-14.346 \\
0.815 \\
0\end{array}$ & $\begin{array}{l}\times 10^{2} \\
5 \\
5\end{array}$ & $\begin{array}{l}-4.37 \times 10^{2} \\
-1.96 \times 10^{3} \\
-0.462 \\
0\end{array}$ & $\left.\begin{array}{l}2.48 \times 10^{6} \\
1.03 \times 10^{7} \\
2.42 \times 10^{3} \\
1\end{array}\right)$ \\
\hline
\end{tabular}

像素-物理全景拼接图显示. 对应的位置数据如表 2 所示, 其中包含单场景下物理坐标及统一坐标系 下坐标. 设空间定位点的实际坐标为 $\left\{L_{i}^{\text {real }} \mid 1 \leqslant i \leqslant 4\right\}$, 则将误差定义为

$$
E_{\text {error }}=\frac{\sum_{i=1}^{4}\left\|L_{i}^{\text {real }}-L_{i}\right\|_{2}}{4 \cdot\left\|L_{\mathrm{s}}\right\|_{2}} \times 100 \%
$$

其中, $\|\cdot\|_{2}$ 符号为 2 个点的欧几里得度量; $L_{\mathrm{s}}$ 为单场 景的有效视野长度 $(40 \mathrm{~m})$ 的一半, 本实验设 $L_{\mathrm{s}}=20 \mathrm{~m}$.

表 2 实验场景下车辆空间定位结果

\begin{tabular}{ccrc}
\hline 定位 & 单场景物理坐标 $/ \mathrm{cm}$ & 统一坐标系坐标 $/ \mathrm{cm}$ & 误差 $/ \%$ \\
\hline$L_{1}$ & $(-393,1285,70)$ & $(136,1234,70)$ & 0.14 \\
$L_{2}$ & $(-341,2096,70)$ & $(84,2038,70)$ & 0.31 \\
$L_{3}$ & $(-337,1090,70)$ & $(112,3132,70)$ & 0.23 \\
$L_{4}$ & $(-323,2683,70)$ & $(97,4725,70)$ & 0.36 \\
\hline
\end{tabular}

由表 2 可以看出, 在近镜头位置处定位较准, 而远离镜头位置定位稍变差, 一是由于镜头边缘
本身存在一定的径向畸变; 再者, 远位置车辆投影 变小, 使 $3 \mathrm{D}$ 包络误差增大. 同时由表 2 可以看出, 由于采取了基于车道线的平移矢量修正，跨场景 后定位误差并没有明显增加.

\section{2 实际场景验证}

为了验证实际场景下的本文方法应用效果， 本文设计了西安市南二环彩虹桥附近的 2 个跨相 机场景, 相比于实验场景, 实际场景有效视野更 广, 达到 100 150 m. 图 8a 和图 8b 所示为通过背 景建模获得的 2 场景背景图, 其中道路虚线长为 $6.0 \mathrm{~m}$, 道路宽为 $7.5 \mathrm{~m}$. 为了获取公共特征点, 本 文利用道路场景中常见的道路虚线标识，观察并 标出公共区域的虚线标识端点并一一对应，同第 3.1 节过程类似, 可解得标定参数、投影矩阵及统 一空间坐标变换矩阵, 如表 3 所示. 并构建跨相机 场景像素-物理全景拼接图, 如图 8c 所示, 由于此 场景视野过长, 限于页面显示, 本文将其分成 2 段 进行展示.

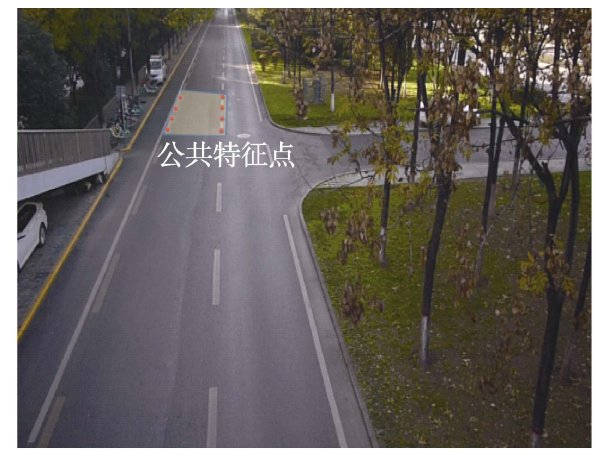

a. 场景 1

$y$
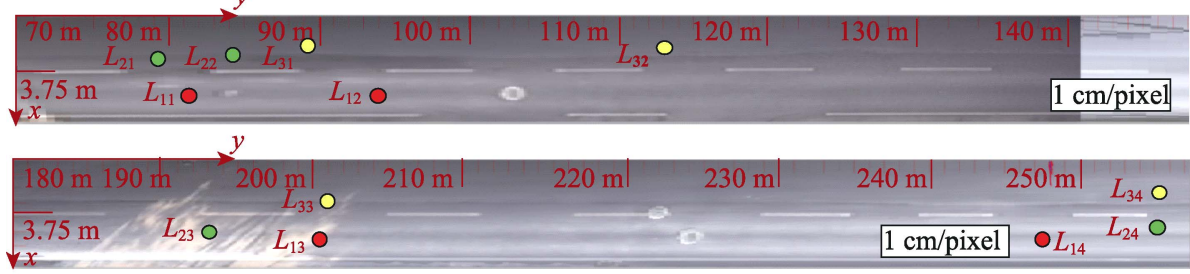

c. 统一坐标系的像素-物理俯视拼接图

图 8 实际场景下跨相机像素-物理全景图拼接结果 
表 3 实际场景下的标定结果及统一空间坐标变换矩阵

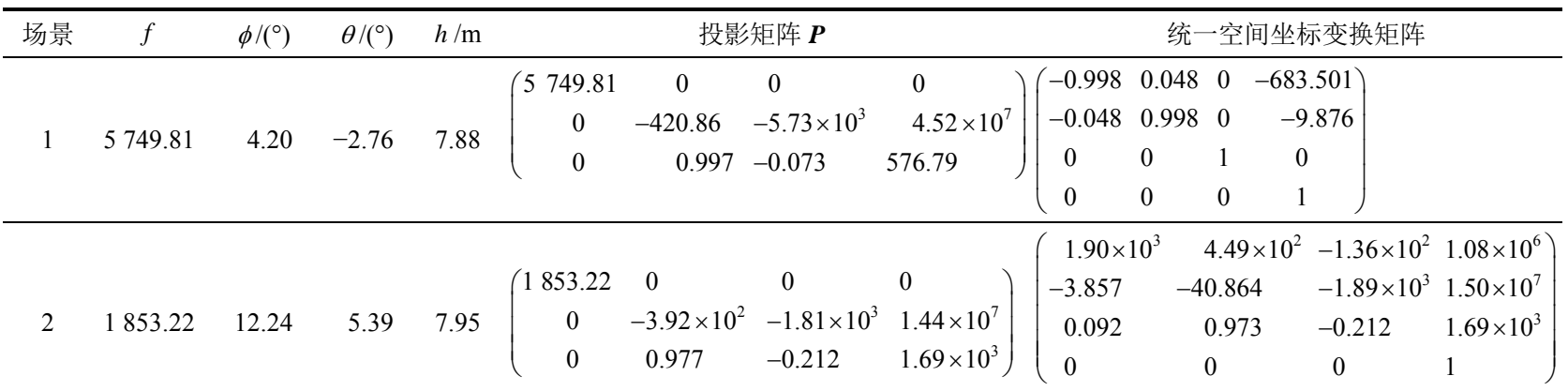

为了说明本文方法的适应性，选取了 3 种不同 类型的车辆, 并对每辆车随机在 2 个场景中各选取 2 个定位进行验证. 与实验场景不同, 所选取车辆
的长宽高已知，因此须利用第 2.2 节提出的投影轮 廓约束方法进行优化估计，最终 3D 包络结果如图 9 所示. 表 4 第 2 列所示为估算出的车辆 3D 尺寸.

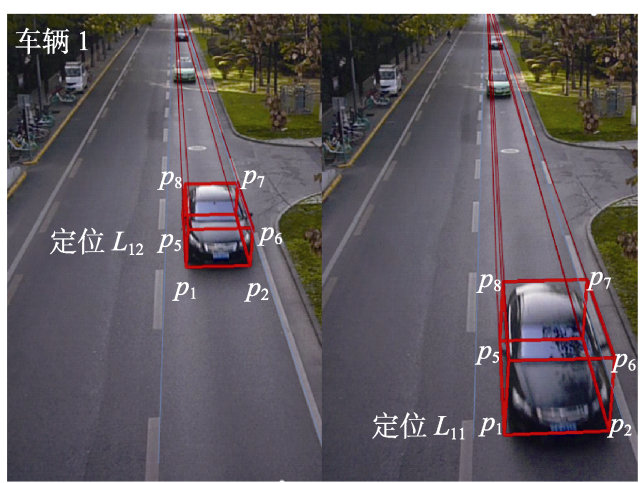

a. 车辆1场景1定位

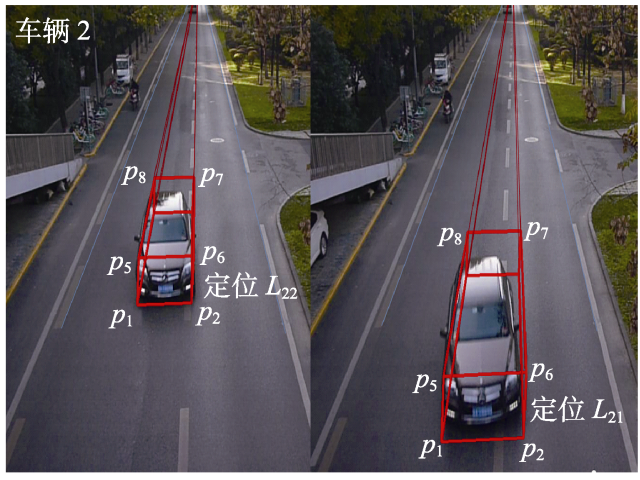

c. 车辆2场景1定位

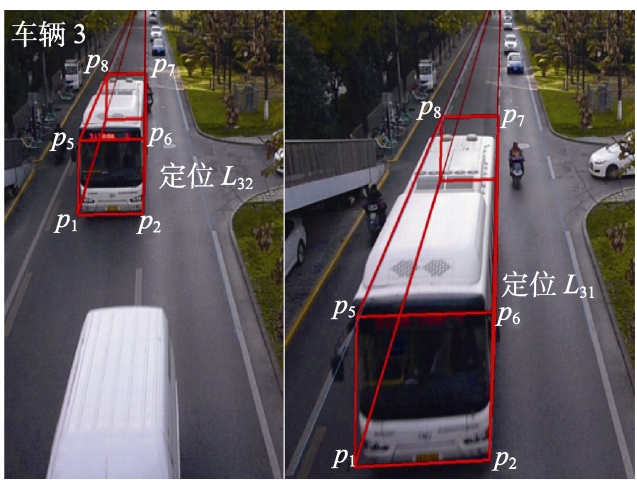

e. 车辆3场景 1 定位

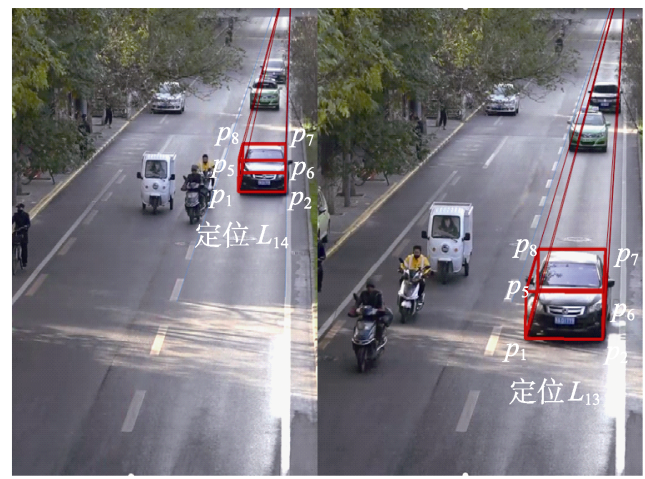

b. 车辆 1 场景 2 定位

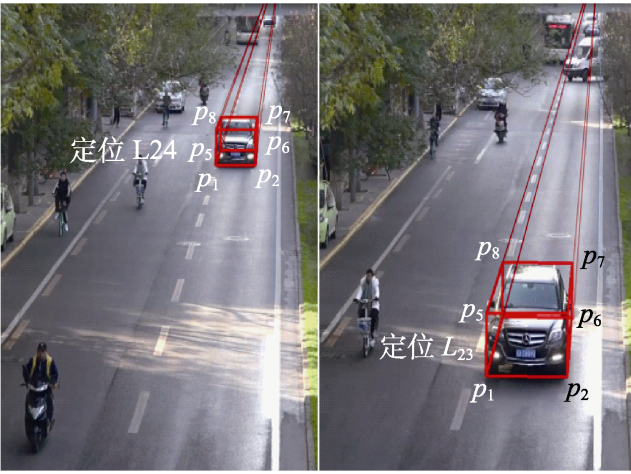

d. 车辆 2 场景 2 定位

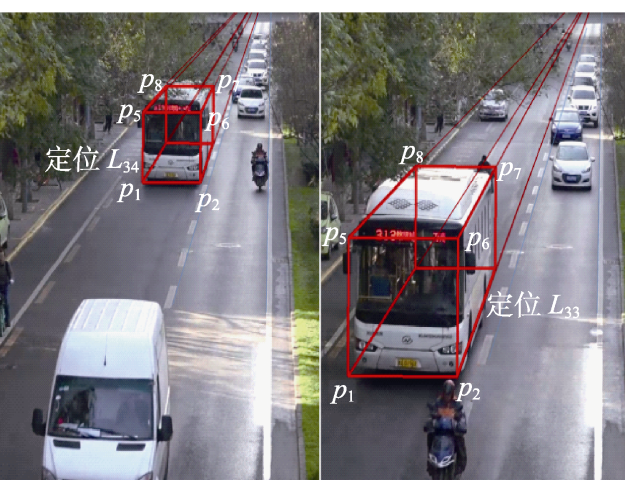

f. 车辆 3 场景 2 定位

图 9 实际场景下车辆 3D 包络结果 
表 4 实际场景下车辆空间定位及 3D 包络结果

\begin{tabular}{|c|c|c|c|c|}
\hline 车辆 & 3D 尺寸估计/cm & & 一坐标系/cm & 平均误差 $/ \%$ \\
\hline \multirow{4}{*}{1} & \multirow{4}{*}{$(425,163,140)$} & $L_{11}$ & $(559,8140,70)$ & \multirow{4}{*}{0.26} \\
\hline & & $L_{12}$ & $(535,9354,70)$ & \\
\hline & & $L_{13}$ & $(548,20092,70)$ & \\
\hline & & $L_{14}$ & $(572,24736,70)$ & \\
\hline \multirow{4}{*}{2} & \multirow{4}{*}{$(524,196,185)$} & $L_{21}$ & $(344,7926,92)$ & \multirow{4}{*}{0.38} \\
\hline & & $L_{22}$ & $(286,8431,92)$ & \\
\hline & & $L_{23}$ & $(492,19293,92)$ & \\
\hline & & $L_{24}$ & $(459,25497,92)$ & \\
\hline \multirow{4}{*}{3} & \multirow{4}{*}{$(1235,250,306)$} & $L_{31}$ & $(191,8911,153)$ & \multirow{4}{*}{1.06} \\
\hline & & $L_{32}$ & $(204,11283,153)$ & \\
\hline & & $L_{33}$ & $(236,20095,153)$ & \\
\hline & & $L_{34}$ & $(220,25549,153)$ & \\
\hline
\end{tabular}

将每个场景中求取的质心变换至统一坐标系, 统 一坐标如表 4 第 3 列所示. 通过记录下车辆的实 际位置, 以及车辆的确切物理尺寸, 求取出其实 际坐标点, 表 4 第 4 列所示为每辆车定位的平均 误差. 为了直观显示, 将每个验证车辆的统一坐 标投影至统一坐标系的像素-物理拼接图, 如图 $8 \mathrm{c}$ 所示, 其中不同的车辆用不同的颜色标注其定 位点.

通过实验结果分析可知，本文方法不论在实 验验证场景还是实际应用场景下均取得了较好的 效果，通过分析发现，在 $20 \sim 40 \mathrm{~m}$ 的实验场景视野 下，定位精度达到厘米级，在 $100 \sim 150 \mathrm{~m}$ 实际场景 视野下的精度也可达到分米级. 通过后续大量实 验表明，在实际应用环境下平均定位精度小于 $0.1 \mathrm{~m}$. 对于无人驾驶的应用精度略低, 但定位参 数对于后续智能交通及车路协同的应用具有较大 价值，同时可对全场景桥梁荷载模型检测提供重 要的数据支撑. 此外, 由于本文对于车辆 $3 \mathrm{D}$ 形态 识别采用的是几何约束估计的方法，因此，整体定 位速度较快, 在已知车辆物理尺寸下单车定位时 间小于 $100 \mathrm{~ms}$, 而在车辆尺寸未知的情况下, 由于 需要优化估计车辆 $3 \mathrm{D}$ 包络, 因此单车位置估计在 $200 \mathrm{~ms}$ 左右，整体可做到实时应用.

然而，通过实验分析也发现本文方法具有的 限制. 在相机偏转角度过小时, 车辆侧身投影信息 损失较多, 造成 $3 \mathrm{D}$ 包络精度降低, 进而造成定位 精度下降, 如图 9 中定位 $L_{21}$ 与 $L_{31}$ 所示; 在画幅的 边缘处, 一是由于车辆投影小, 造成包络的扰动较 大, 二是由于相机本身具有径向畸变, 也会造成定 位精度降低. 综上所述, 在后期的工作中可以继续 改进方法以适应复杂的应用场景，或在实际应用
中可适当调大相机偏转角, 同时使定位车辆尽量 处于非画幅边缘位置.

\section{4 结 语}

通过实验验证，在实验和实际跨相机验证场 景下，本文提出的跨相机场景下的车辆空间定位 方法对于多类型的车辆空间定位估计均取得了较 好的结果. 本文方法的主要贡献有：(1) 构建了基 于相机自标定空间模型的全场景统一坐标系, 使 多场景空间点可便捷地转换至道路统一坐标系中, 并提出了全场景的像素-物理拼接图构建方法, 可 直观地显示多场景中的空间定位点及相互关系; (2) 针对单目视觉下的车辆 $3 \mathrm{D}$ 包络及质心定位难 点, 对于已知物理尺寸的车辆可根据灭点几何约 束快速构建其 3D 包络, 而对于未知几何尺寸的车 辆, 也可根据车辆投影轮廓进行约束优化, 估算出 其 $3 \mathrm{D}$ 包络. 最终可形成一套跨相机全场景的车辆 空间定位系统, 可为智能交通、车路协同及桥梁荷 载模型等后续应用提供重要的数据支撑.

由于在未知车辆物理尺寸的条件下，本文方 法构建车辆 $3 \mathrm{D}$ 包络对于相机偏转角比较敏感, 后 续工作考虑引人较精细化的深度网络对车型进行 识别, 以获取其较为准确的初始物理尺寸. 同时, 本文方法建立在可构建统一坐标系基础之上，而 统一坐标系的构建需要具有已知的公共区域特征 点, 但也存在于实际应用中, 跨相机间不存在公共 特征点或特征点过少, 无法直接构建精确的相邻 场景空间坐标系的变换关系, 此类问题也是后续 需要着重解决的难点.

\section{参考文献(References):}

[1] Novak L. Vehicle detection and pose estimation for autonomous driving[D]. Prague: Czech Technical University in Prague, 2017

[2] Gupta I, Rangesh A, Trivedi M. 3D bounding boxes for road vehicles: a one-stage, localization prioritized approach using single monocular images[C]//Proceedings of the European Conference on Computer Vision. Heidelberg: Springer, 2018: 626-641

[3] He H S, Shao Z Z, Tan J D. Recognition of car makes and models from a single traffic-camera image[J]. IEEE Transactions on Intelligent Transportation Systems, 2015, 16(6): 3182-3192

[4] Lan J H, Li J, Hu G D, et al. Vehicle speed measurement based on gray constraint optical flow algorithm[J]. Optik, 2014, 125(1): 289-295 
[5] Shi S S, Wang X G, Li H S. PointRCNN: 3D object proposal generation and detection from point cloud[C] //Proceedings of the IEEE Conference on Computer Vision and Pattern Recognition. Los Alamitos: IEEE Computer Society Press, 2019: 770-779

[6] Cornick M T, Koechling J C, Stanley B, et al. Localizing ground penetrating RADAR: a step toward robust autonomous ground vehicle localization[J]. Journal of Field Robotics, 2016, 33(1): 82-102

[7] Lee B H, Song J H, Im J H, et al. GPS/DR error estimation for autonomous vehicle localization[J]. Sensors, 2015, 15(8): 20779-20798

[8] Brown M, Lowe D G. Automatic panoramic image stitching using invariant features[J]. International Journal of Computer Vision, 2007, 74(1): 59-73

[9] Dai Huayang, Ran Feipeng. Fast panorama stitching algorithm for mobile devices[J]. Journal of Computer Applications, 2014, 34(9):2673-2677(in Chinese)

(戴华阳，手飞鹏. 一种适于移动设备的全景图快速拼接 方法[J]. 计算机应用, 2014, 34(9): 2673-2677)

[10] Wan Yuandong. Multi-camera calibration and image mosaic method for detecting parts[D]. Wuhan: Huazhong University of Science and Technology, 2017(in Chinese) (万袁冬. 用于零件检测的多相机标定与图像拼接方法研 究[D]. 武汉: 华中科技大学, 2017)

[11] Wang F B, Tu P, Wu C, et al. Multi-image mosaic with SIFT and vision measurement for microscale structures processed by femtosecond laser[J]. Optics and Lasers in Engineering, 2018, 100(1): 124-130

[12] Wu Feifan, Liang Haoxiang, Song Huansheng, et al. Multi-camera traffic scene mosaic based on camera calibration[J]. Computer Systems \& Applications, 2020, 29(1): 176-183(in Chinese)

(武非凡，梁浩翔，宋焕生，等. 基于相机标定的跨相机 场景拼接方法[J]. 计算机系统应用, 2020, 29(1): 176-183)

[13] Caetano T S, Caelli T, Schuurmans D, et al. Graphical models and point pattern matching[J]. IEEE Transactions on Pattern Analysis and Machine Intelligence, 2006, 28(10): 1646-1663

[14] Qu H B, Wang J Q, Li B, et al. Probabilistic model for robust affine and non-rigid point set matching[J]. IEEE Transactions on Pattern Analysis and Machine Intelligence, 2017, 39(2): 371-384

[15] Zhang Z X, Tan T N, Huang K Q, et al. Three-dimensional deformable-model-based localization and recognition of road vehicles[J]. IEEE Transactions on Image Processing, 2011, 21(1): 1-13

[16] Corral-Soto E R, Elder J H. Slot cars: 3D modelling for improved visual traffic analytics[C] //Proceedings of the
IEEE Conference on Computer Vision and Pattern Recognition Workshops. Los Alamitos: IEEE Computer Society Press, 2017: 889-897

[17] Sochor J, Špaňhel J, Herout A. Boxcars: improving fine-grained recognition of vehicles using 3-D bounding boxes in traffic surveillance[J]. IEEE Transactions on Intelligent Transportation Systems, 2018, 20(1): 97-108

[18] Yang S C, Scherer S. CubeSLAM: monocular 3D object SLAM[J]. IEEE Transactions on Robotics, 2019, 35(4): 925-938

[19] He T, Soatto S. Mono3D++: monocular 3D vehicle detection with two-scale $3 \mathrm{D}$ hypotheses and task priors[C] //Proceedings of the AAAI Conference on Artificial Intelligence. Palo Alto: AAAI Press, 2019, 33: 8409-8416

[20] Qin Z Y, Wang J L, Lu Y. MonoGRNet: a geometric reasoning network for monocular 3D object localization[C] //Proceedings of the AAAI Conference on Artificial Intelligence. Palo Alto: AAAI Press, 2019, 33: 8851-8858

[21] $\mathrm{Xu} \mathrm{B}$, Chen Z Z. Multi-level fusion based 3D object detection from monocular images[C] //Proceedings of the IEEE Conference on Computer Vision and Pattern Recognition. Los Alamitos: IEEE Computer Society Press, 2018: 2345-2353

[22] Mousavian A, Anguelov D, Flynn J, et al. 3D bounding box estimation using deep learning and geometry[C] //Proceedings of the IEEE Conference on Computer Vision and Pattern Recognition. Los Alamitos: IEEE Computer Society Press, 2017: 7074-7082

[23] Wang Wei, Zhang Chaoyang, Tang Xinyao, et al. Automatic self-calibration and optimization algorithm of traffic camera in road scene[J]. Journal of Computer-Aided Design \& Computer Graphics, 2019, 31(11): 1955-1962(in Chinese)

(王伟, 张朝阳, 唐心瑶, 等. 道路场景下相机自动标定 及优化算法 $[\mathrm{J}]$. 计算机辅助设计与图形学学报, 2019 , 31(11): 1955-1962)

[24] Kanhere N K, Birchfield S T. A taxonomy and analysis of camera calibration methods for traffic monitoring applications[J]. IEEE Transactions on Intelligent Transportation Systems, 2010, 11(2): 441-452

[25] Dubská M, Herout A. Real projective plane mapping for detection of orthogonal vanishing points[C] //Proceedings of the 24th British Machine Vision Conference. Guildford: BMVA Press, 2013: 1-10

[26] He K M, Gkioxari G, Dollár P, et al. Mask R-CNN[C] //Proceedings of the IEEE Conference on Computer Vision and Pattern Recognition. Los Alamitos: IEEE Computer Society Press, 2017: 2961-2969 